\title{
Brain imaging: closing the gap between basic research and clinical application is urgently needed
}

\author{
Jan K. Buitelaar · David R. Coghill
}

Published online: 3 December 2013

(c) Springer-Verlag Berlin Heidelberg 2013

Brain imaging has revolutionized neural science over the past decades through the identification and description of the neural circuits involved in social cognition, cognitive control, memory, empathy, mood regulation and many other domains. The characterization of these neural circuits at the level of brain structure and function has deepened our understanding of the neural underpinnings of virtually all psychiatric disorders, from ADHD and autism to anxiety and mood disorders, and from schizophrenia to bipolar illness and Alzheimer's disease. One significant newly acquired insight is that psychiatric disorders may be understood best not so much by a neural lesion model focused on discovering specific brain regions where ADHD, autism and other disorders 'reside'. Rather, a disruption of optimal structural and functional connectivity between separate areas of the brain, alongside interference of well-coordinated and synchronized activity of various neural systems turn out to be a most valuable perspective for understanding the biological basis of psychiatric disorders. These may be conceptualized as 'systems disorders of the brain' [1-5]. Brain imaging has also started to add a developmental perspective by outlining how neural circuits

J. K. Buitelaar $(\bowtie)$

Department of Cognitive Neuroscience, Radboud University Medical Center, Donders Institute for Brain, Cognition and Behaviour, P.O. Box 9101, 6500 HB Nijmegen, The Netherlands e-mail: jan.buitelaar@radboudumc.nl

J. K. Buitelaar

Karakter Child and Adolescent Psychiatry, Nijmegen,

The Netherlands

D. R. Coghill

Division of Neuroscience, Medical Research Institute, University of Dundee, Ninewells Hospital, Dundee, Scotland, UK develop over age and how this influences and interacts with maturation of cognitive functions and development of behavioral competences. As such, developmental psychopathology has been complemented by developmental neuroscience, i.e. the study of individual patterns of development of the structure and functions of the brain. This includes the study of the development of neurochemical systems, myelinization, grey/white matter growth, synaptic function, etc., as well as the study of the genetic and environmental causes of individual variability. Together with progress in identifying genetic risk factors for psychiatric illness, brain imaging, and brain imaging genetics confer the opportunity to provide mechanistic explanations about how genetic and environmental risk factors may affect neural circuits and cognitive systems, and how this may lead to clinical symptoms [6-8].

The current issue provides four review papers on brain imaging that are relevant for child and adolescent psychiatry. Johnston et al. [9] discuss the clinical relevance of brain imaging for clinical prediction in psychiatry. In particular, the application of pattern detection technique such as support vector machines has already demonstrated its potential to predict diagnostic status and might further contribute to predictions on treatment response and course of the disorder. Rubia [10] reviews the functional MRI literature on the functional development of cognitive and motivational control, timing, and attention, as well as the development of resting state neural networks. She signals a shift with age from bottom-up processing regions to topdown control in cortico-cortical and cortico-subcortical pathways. Although psychological trauma is a frequent cause of psychopathology at all ages, research into the neural correlates of psychological trauma in children and adolescents is scarce. Rinne-Albers et al. [11] review the extent literature and conclude that the finding of a smaller 
hippocampal volume as reported in studies in adults with posttraumatic stress disorders (PTSD) is not replicated in pediatric samples. Rather unexpectedly, PTSD in children and adolescents is found to be associated with volumetric abnormalities of the corpus callosum. A fourth paper by Oldehinkel et al. [12] reviews the huge potential of restingstate MRI in describing the functional neural circuits in the brain and of mapping these to psychiatric categories. Resting-state MRI data can be collected rather easily and have special advantages over applying the more classic cognitive activation paradigms in developmental studies that span a wide age range and include subjects with various levels of cognitive skills.

Despite all findings and insights provided by brain imaging, direct clinical applications are as yet few, if any. As it has been phrased, none of these thousands and thousands of brain scans over the past decades have changed how clinicians currently diagnose and treat severe mental illness [13]. Is that a problem? Yes, it is, because ultimately, the added societal value of neuroscience research is based on its translational potential, i.e. the power to bring about new products, algorithms, procedures, and treatments that inform and transform the clinical practice of psychiatry.

What clinical contribution could be made by brain imaging studies? We envisage at least three potential areas of application. First, brain imaging might contribute to the development of new medical and non-medical interventions for psychiatric illness by functional validation at the neural systems levels of risk genes and causal mechanisms [14], by screening potentially new molecules in early stages of drug development for signs of efficacy and safety, and by establishing neural markers that serve as intermediate or substitute outcome of clinical interventions [15]. It might inform and guide clinical decision making in complex diagnostic cases. Secondly, whilst acknowledging that the output of support vector machine algorithms on several parameters of structural MRI data will not replace careful clinical assessment and history taking, an algorithm suggesting an $80 \%$ probability of an autism spectrum disorder case will be relevant input to the clinician who has problems in differentiating autism from developmental language disorder and ADHD [16]. In a similar way, predictions based on resting-state MRI data and diffusiontensor MRI data can provide the clinicians with a brainbased context for decision making.

A third application is the prediction of the course of the disorder over time-persistence versus remission in case of ADHD, single versus multiple episodes in case of depression-and of treatment response, and/or risk for side effects. These predictions can be placed in the context of the clinical need for profiling and staging of psychiatric disorders [17, 18]. Imaging parameters could be used as biomarkers to profile clinical heterogeneous disorders into biologically more homogeneous subgroups. For example, into more executive function deficit, reward, or timing deficit type of ADHD [19]. Dissecting the extent and localization of abnormalities in the fronto-striatal circuits may assist dissecting ADHD into rather uncomplicated forms of the disorder, and forms with further escalation into substance use disorder [20].

Of course, implementation of functional or structural brain measures for clinical prediction is not a goal per se. Rather, it should be shown to add significantly to prediction from clinical variables, and be feasible and cost-effective. This requirement is not superfluous, as was demonstrated by the ADHD 200 competition where prediction on the basis of personal characteristics outperformed the prediction on the basis of resting-state MRI data [21].

There are several explanations for the existence of this translational gap. The first is that patients participating in brain imaging studies are, in general, not at all representative of the patients in the real world of daily clinical practice. For the typical brain imaging study, participants have to satisfy strict inclusion and exclusion criteria, and this often leads to exclusion of the most severe patients who present with the highest levels of anxiety, restlessness, hyperactivity, mania, and paranoia. By the same logic, participants with complicating comorbid disorders tend to be excluded. There are also indications for age bias. Thus, cognitive activation studies in autism have been focused strongly on adults rather than on children and adolescents with the disorder [22]. There are hardly any imaging studies in subjects with more severe autism, in spite of the fact about $40 \%$ of all subject with autism are low functioning [23].

A second issue is that successful translation asks for collection of different data and probably also a different type of researcher. Brain imaging databases have to be enriched with standardized and reliable information about the development of symptoms and impairment over time, the type and intensity/dosage of medical and non-medical interventions, response to treatment, and assessment of side effects and complications of treatment. Designing and conducting these brain imaging studies type 2.0 requires input from clinical researchers and neuroscientists with a clinical interest and a keen eye toward translational potential and valorization. Scanning time and procedures may need to be compromised or spread over time and a basic set of structural, diffusion weighted and resting-state MRI scans acquired. The latter may suffice to capture more than $90 \%$ of the relevant information about neural networks that is provided by more complicated and time consuming activation paradigms [24].

There is an urgent need to close the gap between basic brain imaging studies and clinical practice, and move clinical—child and adolescent-psychiatry into clinical neuroscience. 


\section{References}

1. Konrad K, Eickhoff SB (2010) Is the ADHD brain wired differently? A review on structural and functional connectivity in attention deficit hyperactivity disorder. Hum Brain Mapp 31(6):904-916

2. Wass S (2011) Distortions and disconnections: disrupted brain connectivity in autism. Brain Cogn 75(1):18-28

3. van Ewijk H, Heslenfeld DJ, Zwiers MP, Buitelaar JK, Oosterlaan J (2012) Diffusion tensor imaging in attention deficit/ hyperactivity disorder: a systematic review and meta-analysis. Neurosci Biobehav Rev 36(4):1093-1106

4. Deshpande G, Libero LE, Sreenivasan KR, Deshpande HD, Kana RK (2013) Identification of neural connectivity signatures of autism using machine learning. Front Hum Neurosci. 7:670. doi:10.3389/fnhum.2013.00670

5. Vissers ME, Cohen MX, Geurts HM (2012) Brain connectivity and high functioning autism: a promising path of research that needs refined models, methodological convergence, and stronger behavioral links. Neurosci Biobehav Rev 36(1):604-625

6. Munafò MR, Brown SM, Hariri AR (2008) Serotonin transporter (5-HTTLPR) genotype and amygdala activation: a meta-analysis. Biol Psychiatry. 63(9):852-857 Erratum in: Biol Psychiatry 2009 Aug 1;66(3):302

7. Heinz A, Smolka MN, Braus DF, Wrase J, Beck A, Flor H, Mann K, Schumann G, Büchel C, Hariri AR, Weinberger DR (2007) Serotonin transporter genotype (5-HTTLPR): effects of neutral and undefined conditions on amygdala activation. Biol Psychiatry 61(8):1011-1014

8. Mier D, Kirsch P, Meyer-Lindenberg A (2010) Neural substrates of pleiotropic action of genetic variation in COMT: a meta-analysis. Mol Psychiatry. 15(9):918-927

9. Johnston BA, Mwangi B, Matthews K, Coghill D, Steele JD (2013) Predictive classification of individual magnetic resonance imaging scans from children and adolescents. Eur Child and Adolescent Psychiatry (this issue). doi:10.1007/s00787-0120319-0

10. Rubia K (2013) Functional brain imaging across development. Eur Child Adolescent Psychiatry (this issue). doi:10.1007/ s00787-012-0291-8

11. Rinne-Albers MAW, van der Wee NJA, Lamers-Winkelman F, Vermeiren RRJM (2013) Neuroimaging in children, adolescents and young adults with psychological trauma. Eur Child Adolescent Psychiatry (this issue). doi:10.1007/s00787-013-0410-1

12. Oldehinkel M, Francx W, Beckmann CF, Buitelaar JK, Mennes M: Resting-state FMRI research in child psychiatric disorders. Eur Child Adolescent Psychiatry (this issue). doi:10.1007/ s00787-013-0480-0
13. Insel TR (2011) A bridge to somewhere. Transl Psychiatry. 1:e2. doi:10.1038/tp.2011.4

14. Vorstman J, Spooren W, Persico A, Collier D, Aigner S, Jagasia R, Glennon J, Buitelaar J (2013) Using genetic findings in autism for the development of new pharmaceutical compounds. Psychopharmacology (in press)

15. Ecker C, Spooren W, Murphy DG (2013) Translational approaches to the biology of Autism: false dawn or a new era? Mol Psychiatry. 18(4):435-442

16. Ecker C, Rocha-Rego V, Johnston P, Mourao-Miranda J, Marquand A, Daly EM, Brammer MJ, Murphy C, Murphy DG (2010) MRC AIMS consortium. Investigating the predictive value of whole-brain structural MR scans in autism: a pattern classification approach. Neuroimage 49(1):44-56

17. McGorry PD (2013) Early clinical phenotypes, clinical staging, and strategic biomarker research: building blocks for personalized psychiatry. Biol Psychiatry 74(6):394-395

18. Wood SJ, Yung AR, McGorry PD, Pantelis C (2011) Neuroimaging and treatment evidence for clinical staging in psychotic disorders: from the at-risk mental state to chronic schizophrenia. Biol Psychiatry 70(7):619-625

19. Durston S, van Belle J, de Zeeuw P (2011) Differentiating frontostriatal and fronto-cerebellar circuits in attention-deficit/ hyperactivity disorder. Biol Psychiatry 69(12):1178-1184

20. George O, Koob GF (2010) Individual differences in prefrontal cortex function and the transition from drug use to drug dependence. Neurosci Biobehav Rev 35(2):232-247

21. Brown MR, Sidhu GS, Greiner R, Asgarian N, Bastani M, Silverstone PH, Greenshaw AJ, Dursun SM (2012) ADHD-200 Global competition: diagnosing ADHD using personal characteristic data can outperform resting-state fMRI measurements. Front Syst Neurosci 6:69

22. Philip RC, Dauvermann MR, Whalley HC, Baynham K, Lawrie SM, Stanfield AC (2012) A systematic review and meta-analysis of the fMRI investigation of autism spectrum disorders. Neurosci Biobehav Rev 36(2):901-942

23. Baird G, Simonoff E, Pickles A, Chandler S, Loucas T, Meldrum D, Charman T (2006) Prevalence of disorders of the autism spectrum in a population cohort of children in South Thames: the special needs and autism project (SNAP). Lancet 368(9531):210-215

24. Smith SM, Fox PT, Miller KL, Glahn DC, Fox PM, Mackay CE, Filippini N, Watkins KE, Toro R, Laird AR, Beckmann CF (2009) Correspondence of the brain's functional architecture during activation and rest. Proc Natl Acad Sci USA 106(31):13040-13045 